\title{
Sonomammographic characteristics of invasive lobular carcinoma
}

This article was published in the following Dove Press journal:

Breast Cancer:Targets and Therapy

27 July 2012

Number of times this article has been viewed

\author{
Osama R Kombar ${ }^{1,3}$ \\ Dalia M Fahmy' \\ Mary V Brown ${ }^{3}$ \\ Omar Farouk ${ }^{2}$ \\ Osama El-Damshety ${ }^{2}$ \\ 'Diagnostic Radiology Department, \\ ${ }^{2}$ Surgical Oncology Department, \\ Oncology Center, Mansoura \\ University, Mansoura, Egypt; \\ ${ }^{3}$ Diagnostic Radiology Department, \\ Al-Amiri Hospital, Safat, Kuwait
}

\section{Video abstract}

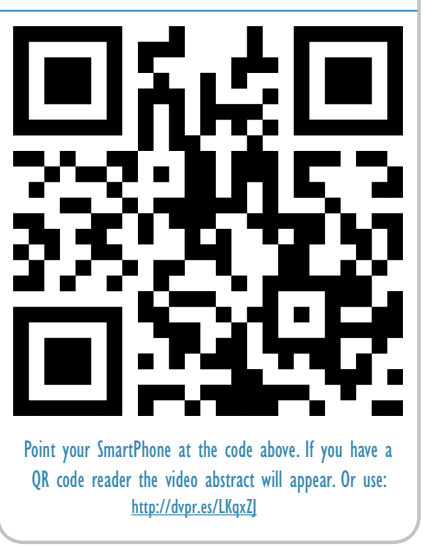

Correspondence: Dalia M Fahmy Block 3, Street 3, Radiology Department, Taiba Hospital, Sabah El-Salem, Kuwait

Tel +96560054578

Fax +9655 513857

Email daliamonir2525@gmail.com
Objective: The objective of our study was to identify characteristic features of invasive lobular carcinoma on mammography and ultrasound examinations

Materials and methods: This is a retrospective multicenter study of women with biopsyproven invasive lobular carcinoma. All patients had undergone diagnostic sonomammography. The imaging findings were identified by experienced breast imagers. Final surgical pathology results were used as the reference standard.

Results: Thirty-two women ranging in age from 42 to 63 years old (mean age, 53 years), All had biopsy-proven invasive lobular carcinomas. Common features on mammogram included dense mass followed by architectural distortion; three cases showed breast asymmetry and one case was reported as normal. On ultrasound, common features included solid mass with spiculated margins, posterior shadowing, and perpendicular to the skin.

Conclusion: Although no specific features could be linked to invasive lobular carcinoma, care should be directed to subtle signs such as architectural distortion and breast asymmetry in order not to miss any lesions. The combination of mammographic and sonographic helps to decrease the relatively high false negative diagnosis of this type of breast cancer.

Keywords: mammography, ultrasound, cancer, breast

\section{Introduction}

The majority of invasive breast carcinomas are categorized as ductal carcinoma of the usual "not otherwise specified" type. Invasive lobular carcinoma (ILC) is the second most common histologic type of breast carcinoma, accounting for approximately $10 \%-15 \%$ of all invasive breast cancers. ${ }^{1}$ Arising from the lobular epithelium, ILC tends to be insidious in onset because it does not invoke a vigorous desmoplastic response. Its noncohesive histologic growth pattern, low likelihood of producing calcifications, and low opacity may account for the fact that ILC is frequently not apparent with mammography and is difficult to identify clinically. ${ }^{2}$ It is usually detected with mammography at a later stage, increasing the likelihood of large primary lesions and positive node status at biopsy.

The aim of this study is to identify characteristic features of ILC on mammography and ultrasound examinations

\section{Patients and methods Case collection}

Radiographic data were collected (from March 2009 to April 2012) from the radiology department at Al-Amiri Hospital in Kuwait as well as two private clinics in Mansoura, Egypt in this multi-institutional retrospective study. All cases included in this study 
presented with clinical symptoms that were suggestive of breast cancer. They underwent diagnostic sonomammography and were referred for biopsy and pathological analysis.

\section{Radiographic examination}

All the cases underwent diagnostic mammography with complementary sonography. All radiologists who interpreted the examinations were experienced breast imagers, each with a minimum of 5 years of experience interpreting mammogram and breast ultrasound.

Mammography was performed with the equipment available at each institution (digital mammography machines: Lorad Selenia and soft copy work station, 2005, Lorad-Hologic, St Petersburg, FL. DMR and Diamond Analog, GE Healthcare, Milwaukee, WI). Sonography was performed using a highfrequency transducer $(10 \mathrm{MHz})$ with the equipment available at each institution (Logic 5 and Voluson e 730; GE Healthcare, Milwaukee, WI. US HDI 4000, A3300520; Philips, SzabolcsSzatmar-Bereg, Hungary).

\section{Histopathological analysis}

All cases underwent histopathological analysis after ultrasound-guided core needle biopsy (true cut). The cases performed in Kuwait underwent biopsies in Al-Amiri Hospital by an experienced radiologist. Cases in Egypt were referred to the oncology center of Mansoura University Hospital where biopsies were performed by oncology surgeons.

Pathologists in both institutions sharing in this study used E-cadherin testing in diagnosis and classification of ILC, which is based on specific criteria and the perceived "aggressiveness" of the cancer. Yet it was difficult to correlate specific radiologic features to each grade due to the limited number of cases included in the study.

\section{Results}

Thirty-two women ranging in age from 42 to 63 years (mean age, 53 years) with biopsy-proven ILCs participated in this study. Only one case had breast asymmetry on mammography only and no masses were detected on ultrasound, yet the surgeon felt a hard lesion and performed a biopsy, which revealed ILC. Another case was reported as benign fibroadenoma on sonomammography, but true-cut biopsy and the final postoperative diagnosis revealed ILC. Sensitivity for combined sonomammography was $94 \%$.

\section{On mammogram}

Twelve cases out of 32 showed architectural distortion (38\%); four of these cases had discrete masses, while the remaining eight cases showed architectural distortion only (Figure 1). Three cases showed breast asymmetry (Figure 2). Twenty-one cases showed single dense masses: six cases had multifocal/multicentric masses (four cases with two masses and two cases with three masses), and 15 had single masses. Twenty-five masses showed irregular speculated margins (Figures 3 and 4), four masses showed smooth margins, and eight masses showed pleomorphic calcifications (Figure 5). Five cases showed skin thickening (Figure 5). Twenty cases showed associated enlarged axillary lymph nodes on mammogram films, yet only seven cases showed loss of fatty hilum. Mammographic findings are summarized in Table 1.

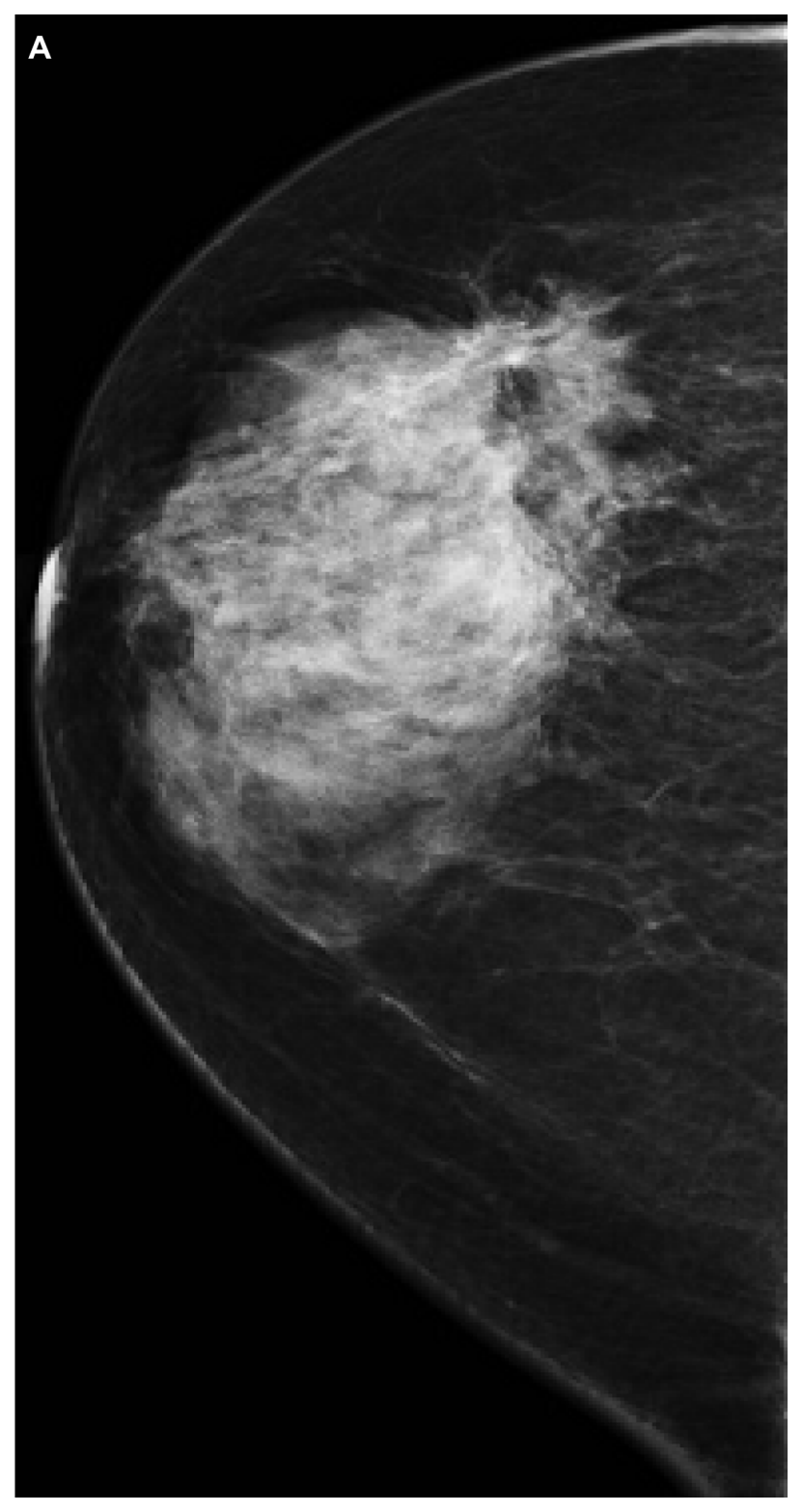

Figure I (Continued) 

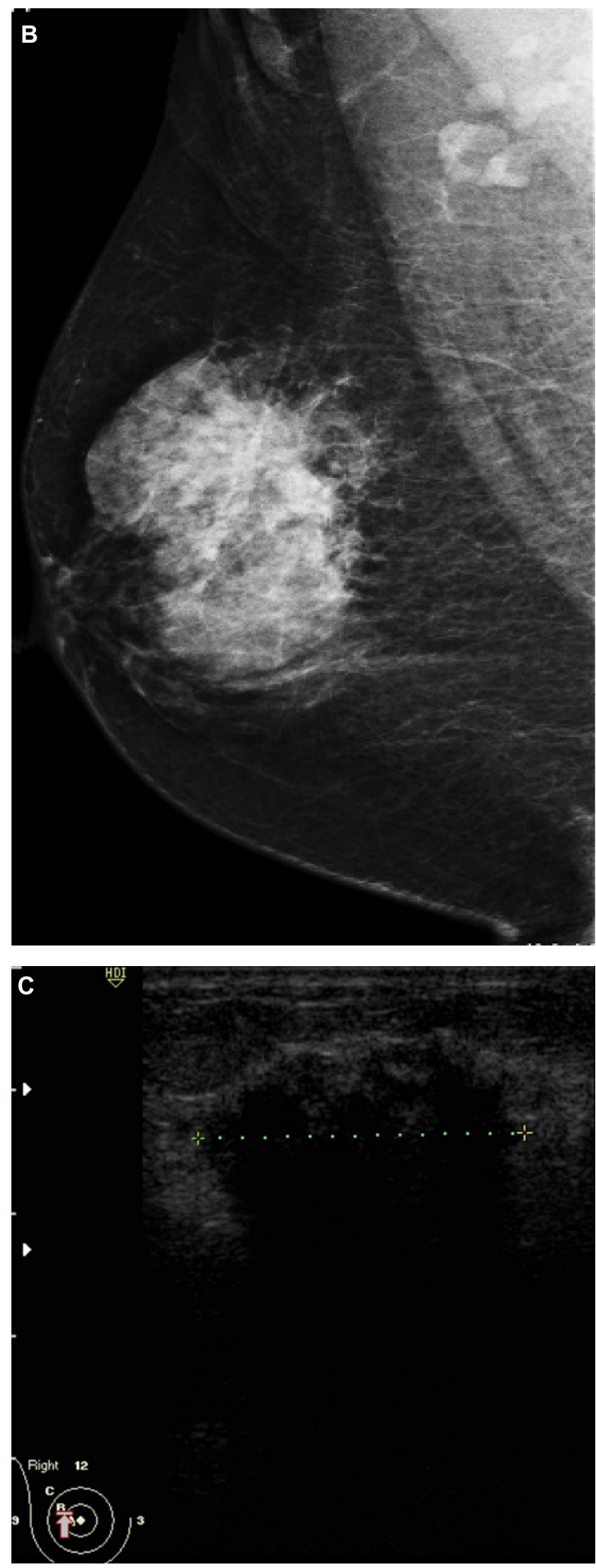

Figure I A 5 I-year-old female presented with hard right breast after suffering from right breast trauma 2 months previously. (A and B) Craniocaudal and mediolateral mammogram of the right breast revealed a dense breast with architectural distortion noted at upper outer quadrant (more evident on craniocaudal view). (C) Ultrasound revealed an ill-defined irregular hypoechoic mass with posterior acoustic shadowing at 9 o'clock of the right breast.

Note: The longitudinal axis of the mass was parallel to that of the skin.
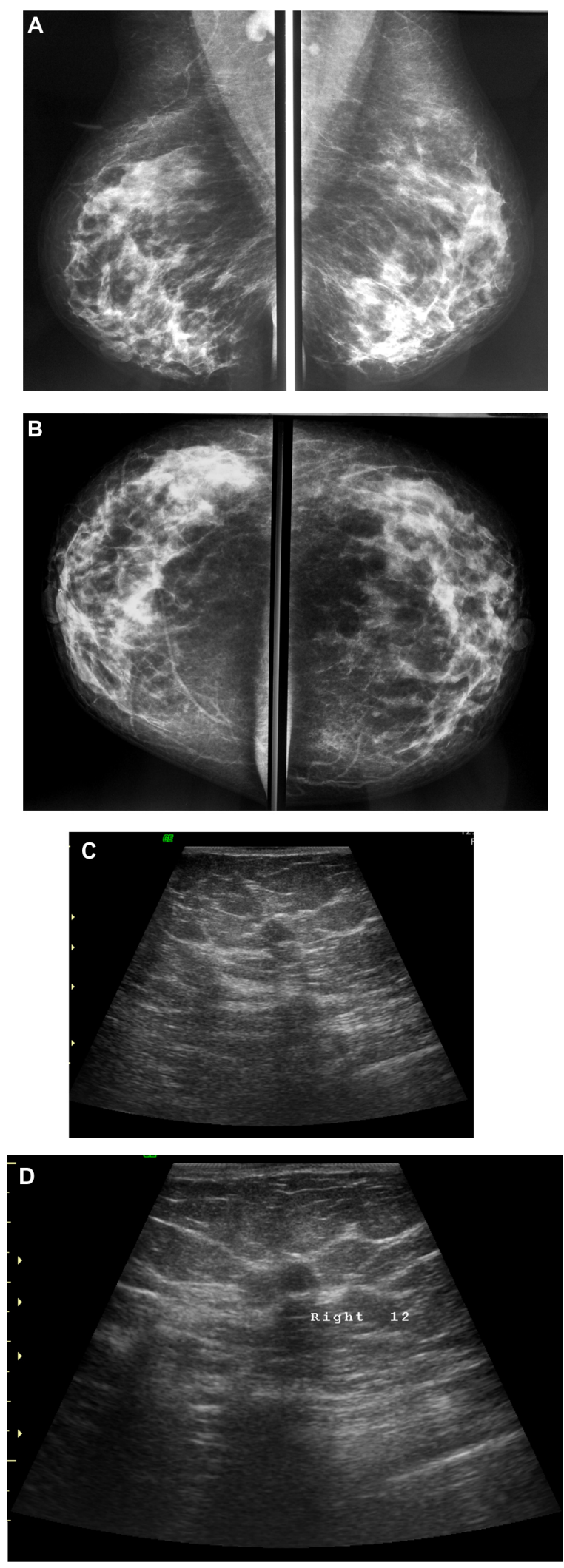

Figure 2 A 48-year-old female patient presented with palpable right axillary lymph node. (A and $\mathbf{B}$ ) Craniocaudal and mediolateral mammogram revealed asymmetric breast density with no definite masses. (C and $\mathbf{D})$ Ultrasound revealed three small hypoechoic masses at I-II o'clock of the right breast (only two are displayed here). Note: The masses have smooth margins, posterior acoustic shadowing, and their long axis was parallel to that of the skin. 

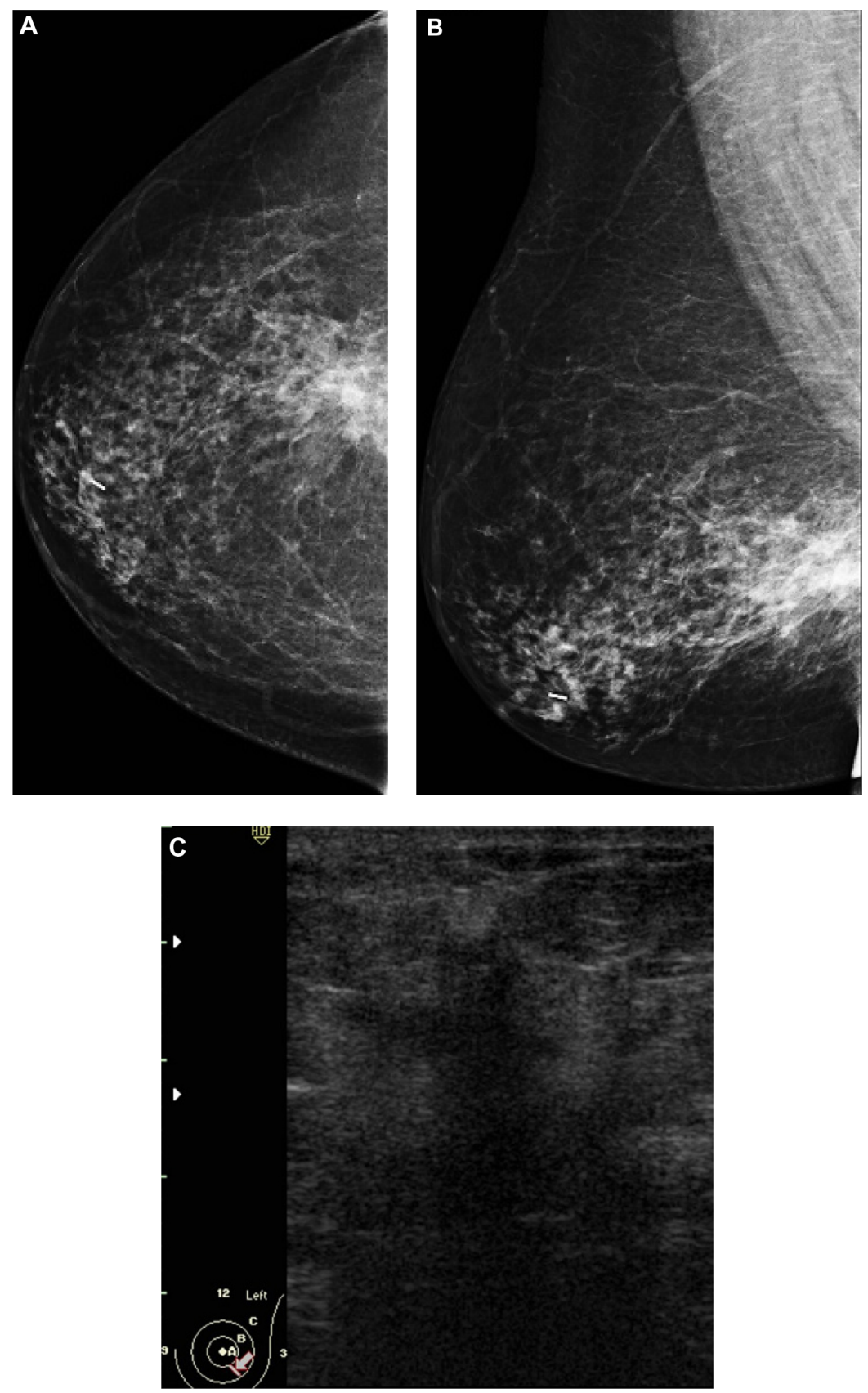

Figure 3 A 58-year-old female with palpable mass in the left breast. (A and B) CC and ML mammogram films revealed ill-defined dense opacity with spiculated margins surrounded by architectural distortion at the lower central area of the breast, which looks to be attached to the pectoralis muscle posteriorly. (C) On ultrasound, ill-defined, spiculated, hypoechoic, solid mass with posterior shadowing measuring $16 \times 13 \mathrm{~mm}$ is seen at 5 o'clock of the breast.

Notes: The mass was inseparable from the underlying muscle. Another smaller, similar hypoechoic mass is seen close by measuring $6 \times 5$ mm.

Abbreviations: CC, craniocaudal; ML, mediolateral.

\section{On ultrasonography}

Thirty-five solid masses were detected. Eight cases had multifocal/multicentric masses (three cases had three masses and five had two masses). While six cases did not reveal any mass (architectural distortion only), one case showed only focal shadowing and one case was reported as normal on ultrasound. Twenty-seven masses were hypoechoic with eight of mixed heterogeneous echopattern.
Twenty-eight masses showed irregular infiltrating margins and seven masses showed smooth margins. Twenty-three masses showed posterior shadowing, while 11 masses had no shadowing, and one mass showed posterior acoustic enhancement. Twenty-six masses had long axes perpendicular to that of the skin, while nine masses were parallel to the long axis of the skin. Associated fibroadenosis was detected in 10 cases, duct dilatation in three cases, and 

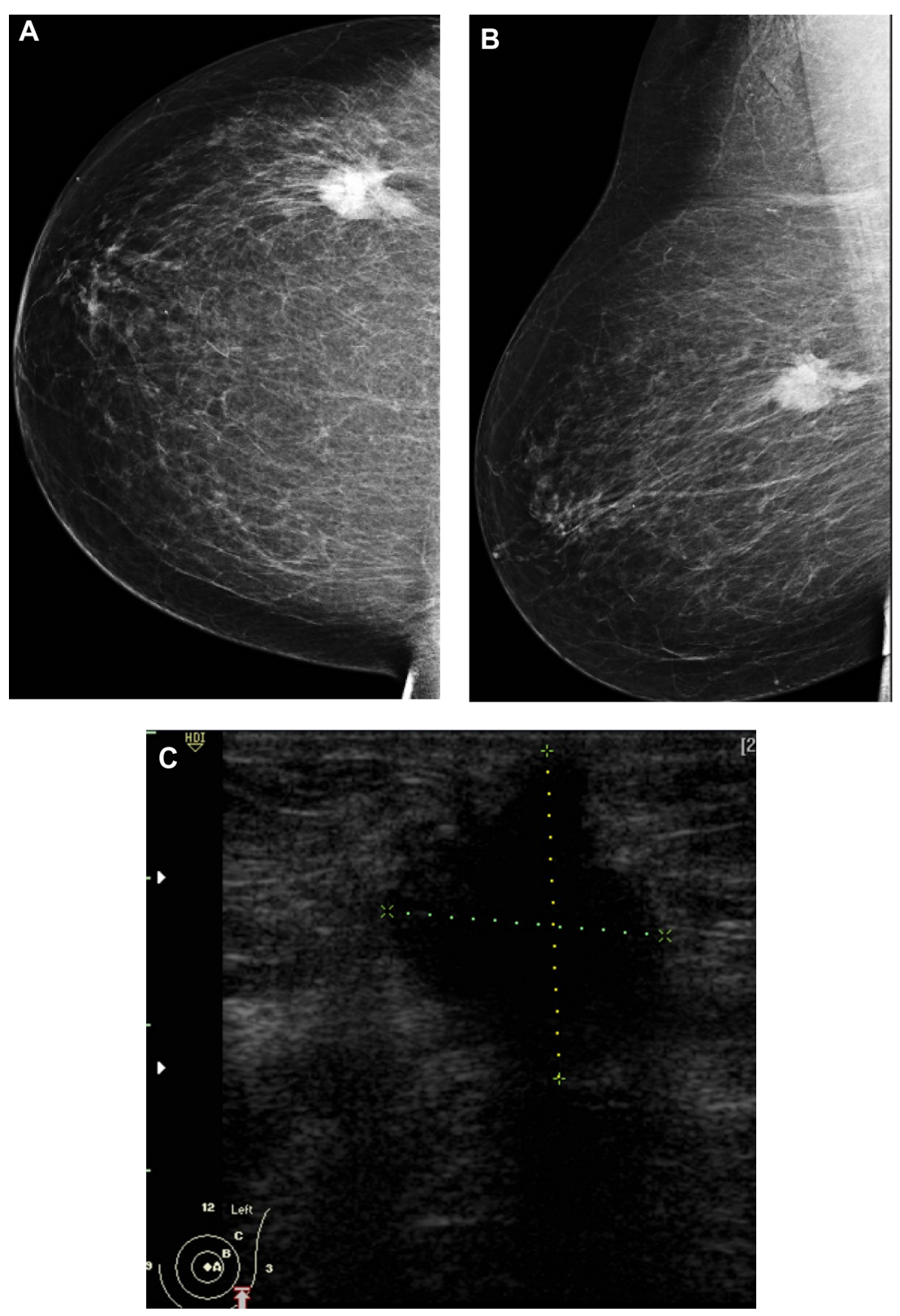

Figure 4 A 72-year-old female with left breast mass. (A and B) Craniocaudal and mediolateral mammogram of left breast revealed an irregular spiculated, dense mass $(35 \times 25 \mathrm{~mm})$ in the upper outer quadrant close to the pectoral muscle. Single benign macrocalcification is noted in the central area. (C) Ultrasound of the left breast revealed a spiculated, hypoechoic, solid mass $(30 \times 23 \mathrm{~mm})$ with strong acoustic shadow at 3-4 o'clock.

Note: The mass extends to the pectoralis muscle with its long axis perpendicular to the skin.

Abbreviations: CC, craniocaudal; ML, mediolateral.

skin thickening in five cases. The sonographic findings are summarized in Table 2.

\section{Discussion}

Mammography has been the gold standard for detecting breast cancer. It is an anatomic approach, relying on the relative densities of breast tissues to differentiate normal breast tissue from breast cancer. The sensitivity of mammography is limited, with an overall sensitivity of $85 \%$, which decreases to $68 \%$ in women with dense breasts. ${ }^{3}$
Furthermore, mammography has relatively lower sensitivity for the detection of ILC compared with the other types of breast cancers. False-negative rates of up to $19 \%$ have been reported for ILC. ${ }^{4}$ This is attributed to a combination of some factors. ILC tends to infiltrate into the surrounding stroma in rows of single cells, with little disruption of the underlying tissues, which makes it less likely than other types of cancers to form discrete masses. ${ }^{5}$ Subtle mammographic features, such as asymmetric densities and architectural distortion, tend to be more common in ILC than in invasive ductal carcinoma (IDC). ${ }^{6}$ 

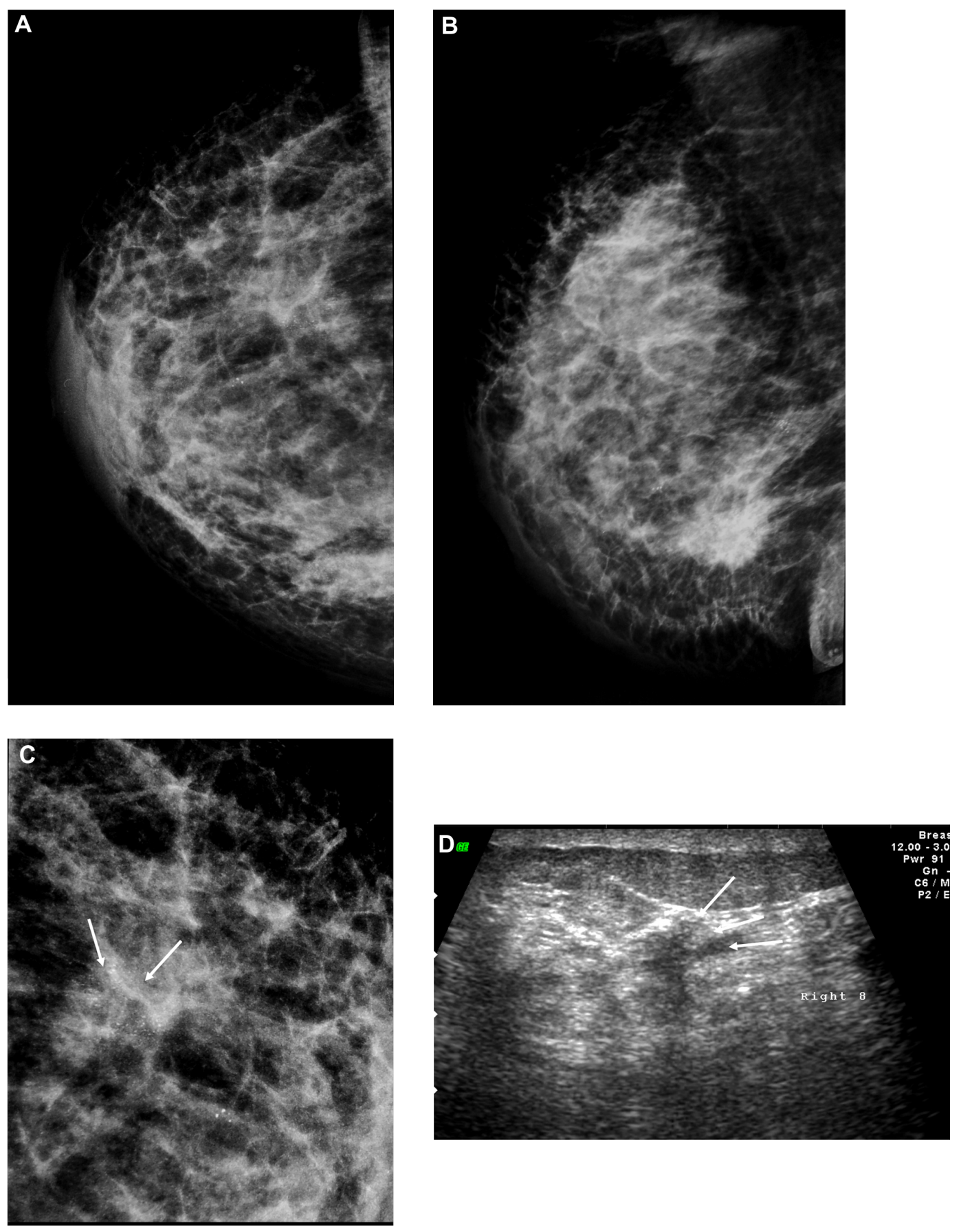

Figure 5 A 48-year-old female patient presented with hard right breast mass of 2 months duration. (A-C) CC, ML, and magnified mammogram views of the right breast revealed an irregular spiculated dense mass at the lower outer quadrant of the right breast. A magnified view shows clusters of pleomorphic microcalcifications within the lesion (arrows). Four foci of calcifications are seen away from the lesion. (D) Ultrasound revealed an ill-defined mass of heterogeneous echopattern at 8 o'clock of left breast.

Note: The mass shows foci of microcalcifications (arrows).

Abbreviations: CC, craniocaudal; ML, mediolateral.

In the current study, eight cases showed architectural distortion without masses on mammography $(25 \%)$, three cases presented as breast asymmetry $(9 \%)$, and 21 cases had discrete masses (66\%). This compares with other researchers who reported architectural distortion as the second most common manifestation of ILC at mammography. ${ }^{7}$ In our study, architectural distortion was seen in $20 \%$ of cases. While asymmetries were found in $18 \%$ and normal or benign findings in $10 \%$ of our cases, discrete masses were found in $45 \%$ of the ILC cases. ${ }^{7}$

We found that $83 \%(n=25)$ of masses had irregular spiculated margins while $17 \%(n=8)$ had smooth margins. 
Table I Mammographic findings of 32 cases of invasive lobular carcinoma

\begin{tabular}{lll}
\hline Feature & $\begin{array}{l}\text { Number of } \\
\text { cases/masses }\end{array}$ & Notes \\
\hline Density & 21 & Total number of \\
Mass & 15 & $\begin{array}{l}\text { single masses }=30 \\
\text { Single }\end{array} \quad$ Four cases had \\
$\quad$ Multifocal/multicentric & 6 & architectural \\
$\quad$ dilateral & 0 & distortion associated \\
Asymmetry & 3 & with mass \\
Architectural distortion & 12 & \\
Size & & \\
$<$ I cm & 3 & \\
I-3 cm & 21 & \\
$>3$ cm & 5 & \\
Margins & & \\
Speculated & 25 & \\
Smooth & 4 & \\
Calcifications & & \\
Microcalcifications & 8 & \\
Macrocalcifications & 1 & \\
Skin thickening & 5 & \\
Nipple retraction & None & \\
\hline
\end{tabular}

Table 2 Sonographic findings in 32 cases of invasive lobular carcinoma

\begin{tabular}{|c|c|c|}
\hline Feature & $\begin{array}{l}\text { Number of } \\
\text { cases/masses }\end{array}$ & Notes \\
\hline Masses & 24 & Total number \\
\hline Single & 16 & of masses $=35$ \\
\hline Multifocal/multicentric & 8 & One case was \\
\hline Bilateral & 0 & reported normal \\
\hline Focal shadowing without mass & I & on ultrasound \\
\hline Architectural distortion & 6 & \\
\hline \multicolumn{3}{|l|}{ Size } \\
\hline$<\mathrm{l} \mathrm{cm}$ & 7 & \\
\hline $\mathrm{I}-3 \mathrm{~cm}$ & 25 & \\
\hline$>3 \mathrm{~cm}$ & 3 & \\
\hline \multicolumn{3}{|l|}{ Mass } \\
\hline Hypoechoic & 27 & \\
\hline Mixed heterogeneous & 8 & \\
\hline \multicolumn{3}{|l|}{ Margins } \\
\hline Irregular, infiltrating & 28 & \\
\hline Rounded, smooth & 7 & \\
\hline \multicolumn{3}{|l|}{ Calcifications } \\
\hline Microcalcifications & 5 & \\
\hline \multicolumn{3}{|l|}{ Posterior acoustic features } \\
\hline Shadowing & 23 & \\
\hline No shadowing & 11 & \\
\hline Acoustic enhancement & I & \\
\hline \multicolumn{3}{|l|}{ Longitudinal axis } \\
\hline Perpendicular to skin & 26 & \\
\hline Parallel to skin & 9 & \\
\hline Associated fibroadenosis & 10 & \\
\hline Associated duct dilatation & 3 & \\
\hline Skin thickening & 5 & \\
\hline
\end{tabular}

This coincides with other studies which reported that ILC most commonly manifests as a mass (44\%-65\% of cases), usually with spiculated or ill-defined margins while rounded and circumscribed masses are less common, being seen in only $1 \%-3 \%$ of ILC cases. ${ }^{8}$

Microcalcifications were detected in eight of 32 cases (25\%). This finding is in agreement with Le Gal et al, ${ }^{9}$ who found suspicious microcalcifications in $24 \%$ of pure ILC cases. However, there are great variations in the percentage of infiltrating lobular carcinoma reported to be associated with suspicious calcification. Other researchers reported a percentage of $1 \%-2 \% .^{10,11}$ This discrepancy could be attributed to the higher accuracy of new mammography machines with computer-aided detection. Cornford et al ${ }^{12}$ cited a higher frequency of microcalcifications in $28 \%$ of cases. They included mixed invasive lobular and ductal tumors in the infiltrating lobular carcinomas category, which could account for the difference. Characteristically, the tumor cells are seen to surround the ducts without obstructing them. It is postulated that the lack of ductal invasion or obstruction may explain why microcalcifications are not often seen with these tumors. ${ }^{13}$

The mammographic diagnosis of ILC is also limited by the fact that the density of ILC on mammography is similar to, or less than that of normal glandular tissue, due to the noncohesive nature of the ILC cells. ${ }^{14}$

In the current study we tried to overcome this by using the postprocessing capabilities of digital mammography. After the images were taken, contrast manipulation improved lesion conspicuity. Magnified images allowed better detection of microcalcification clusters and better evaluation of tumor morphology. This is considered an advantage over film mammography, which would have required extra films.

Ultrasonography is complementary to mammography, especially with regard to evaluating dense breasts and masses that are difficult to assess clinically and mammographically. ${ }^{15}$ Ultrasound has an established role in determining if a clinically or mammographically apparent lesion is real or artifactual, and helps to further characterize the lesions by differentiating solid from cystic lesions. ${ }^{5}$ It also provides guidance for interventional procedures performed in breast imaging such as biopsies, aspirations, drainages, and needle localizations. Ultrasound has been shown to provide a more accurate measurement of the size of a mass than mammography or clinical examination. ${ }^{16}$

Current high-frequency transducers are able to demonstrate malignant features such as spiculations and microlobulations, which were not easily seen with the older $7.5-\mathrm{MHz}$ 
transducers. Harmonic imaging may help to detect subtle hypoechoic masses and to accentuate posterior acoustic shadowing. ${ }^{17}$

In this study we used high-frequency ultrasound (10 MHz). However, as mentioned by other researchers, ${ }^{18}$ higher-frequency ultrasound $(13 \mathrm{MHz})$ is a more effective tool in the diagnosis of small tumors with improved diagnostic safety and reduced unnecessary invasive diagnostic procedures. The sensitivity of ultrasound for detection of ILC is greater than that of mammography, with sensitivities ranging from $68 \%$ to $98 \% .^{13}$

The most common ultrasound manifestation of ILC is an irregular or angular mass with hypoechoic and heterogeneous internal echoes, ill-defined or speculated margins, and posterior acoustic shadowing, findings that are seen in $54 \%-61 \%$ of cases. ${ }^{14}$

In the current study, 27 lesions were hypoechoic and eight were of mixed heterogeneous echopattern. Twenty-eight lesions showed irregular infiltrating margins (80\%), while seven lesions showed smooth margins (20\%).

Twenty-three masses were associated with posterior shadowing (66\%), while 11 lesions were without shadowing (34\%). On the other hand, one case showed posterior acoustic enhancement without mass $(3 \%)$. This compares well with Selinko et al, ${ }^{14}$ who described the appearances of 62 cases of pure ILC. The most common sonographic appearance was a hypoechoic mass, associated with posterior acoustic shadowing in 36 masses (58\%), and without posterior acoustic shadowing in 17 masses $(27 \%)$.

In the current study, 26 masses had long axis perpendicular to that of the skin (74\%), while nine lesions were parallel to the long axis of the skin (26\%). This compares with Cawson et al, ${ }^{19}$ who reported that ILC cases were less likely to be tall than wide when compared with IDC cases. They suggested that the flatter shape reflected the morphology of ILC, with the tumor cells spreading in a horizontal fashion along normal tissue planes, unlike IDC, which tends to grow across tissue planes. ${ }^{19}$

Butler et $a l^{6}$ mentioned that the finding of acoustic shadowing without a distinct mass is usually limited to the classic ILC. They also found that classic ILC is reported as normal in $40 \%$ of ultrasound cases. Other researchers reported posterior acoustic shadowing without an associated mass in $9.5 \%-11 \%$ of cases, ${ }^{13,15}$ and found $(2 \%)$ of cases were sonographically occult. ${ }^{13}$

In our study, we had one case of focal shadowing without a mass $(3 \%)$ and only one case was reported as normal $(3 \%)$. This great discrepancy may be attributed to the use of new ultrasound machines with higher sensitivity in lesion detection.

Although none of the lesions included in this study were hyperechoic, other researchers found that ILC could present with hyperechoic lesions and they were described as an atypical finding by Cawson et al. ${ }^{19}$ They found that 21 of 37 (57\%) of ILCs were echogenic and showed that ILCs were nearly 10 times more likely to be hyperechoic when compared with IDCs. Their explanation was that ILC infiltration into the surrounding tissues is suspected to cause an increase in reflective surfaces sonographically, resulting in increased internal echoes, and therefore increasing the overall echogenicity of ILC.

Other researchers correlated the ultrasound appearances of ILC to its various pathological subtypes. They stated that classic ILC tends to manifest as focal shadowing without a discrete mass, whereas pleomorphic type ILC is more typically seen as a shadowing mass. Signet ring, alveolar, and solid subtypes of ILC are more likely to manifest as a lobulated, well-circumscribed mass. ${ }^{6}$ Unfortunately, we could not confirm this as to do so requires a larger number of cases.

Watermann et $\mathrm{al}^{20}$ compared the ultrasound morphology of 69 cases of ILCs with 337 cases of invasive tumors of other histologic differentiation (TOD). An irregular shape was found in $88 \%$ of ILCs, compared with $67 \%$ of TOD. Margins were indistinct in $94 \%$ of ILCs, compared with $76 \%$ of TOD. Posterior shadowing was observed in $84 \%$ of ILCs and 58\% of TOD. Irregular margins, hyperechoic or isoechoic patterns, and architectural distortions were more frequent in ILCs than in TOD. ${ }^{20}$

Although we were not able to get the actual pathological size, we have found that lesions were smaller on ultrasound than in mammography. This could be explained as the size on mammogram represents the tumor and surrounding desmoplastic reaction. Berg and Gilbreath ${ }^{16}$ found that ultrasound provided a more accurate measurement of the size of a mass compared with mammography, and the findings on ultrasound resulted in a change in surgical management in $18 \%$ of the patients.

Ultrasound imaging showed multifocal/multicentric distribution in eight cases (24\%), while mammography could detect only six cases. This agrees with Selinko et al, ${ }^{14}$ who reported that ultrasound was superior to mammography in identifying multicentricity and multifocality in $21 \%$ of cases in their study. ${ }^{13}$

It is a well-established rule in all institutions sharing in this study to examine both whole breasts in order not to miss any multicentric or multifocal masses. Wilkinson et $\mathrm{al}^{21}$ found 
multicentric/multifocal tumors in 35 (34\%) of the 102 cases of breast cancer who underwent bilateral whole breast ultrasound and in $18(15 \%)$ of the 124 control subjects who underwent targeted breast sonography. They concluded that bilateral whole breast sonography increased the preoperative diagnosis of multiple tumors in women who had primary breast cancer, resulting in a change in management in $8 \%$ of cases.

On the other hand, ultrasound was less sensitive in detection of microcalcifications compared to mammography. We detected microcalcifications in five cases, while it was detected in eight cases by mammography. This finding is in agreement with Moon et al, ${ }^{22}$ who depicted $45 \%$ of 100 mammographically detected clustered calcifications on ultrasound.

We had one case that showed only breast asymmetry on mammogram, while three small lesions less than $1 \mathrm{~cm}$ in size were detected on ultrasound. This enhances the value of adding sonography to mammogram as a diagnostic tool and compares well with Berg et al, ${ }^{23}$ who suggested that ultrasound can be particularly helpful in depicting mammographically subtle or occult ILC.

This study had several limitations. We could not correlate the actual pathological specimen size to that reported by ultrasound and mammography. Some patients were referred to adjuvant chemotherapy before surgical excision. Also, we could not correlate radiological features to pathological variants of ILC as this needed a larger number of cases. Some ultrasound machines used were not updated to recent higher frequencies.

\section{Conclusion}

Although no specific features could be linked to ILC, care should be directed to subtle signs such as architectural distortion and breast asymmetry in order not to miss any lesions. Even on ultrasound, masses may appear with smooth margins, be wider than taller, or may lack posterior shadowing. The combination of mammographic and sonographic findings helps to decrease the relatively high false-negative diagnosis of this type of breast cancer.

\section{Disclosure}

The authors report no conflicts of interest in this work.

\section{References}

1. Li CI, Daling JR. Changes in breast cancer incidence rates in the United States by histologic subtype and race/ethnicity, 1995 to 2004. Cancer Epidemiol Biomarkers Prev. 2007;16:2773-2780.

2. Kopans DB. Malignant lesions of the lobule. In: Kopans DB, editor. Breast Imaging. Baltimore, MD: Lippincott Williams \& Wilkins; 2007:866-870.
3. Rosenberg RD, Hunt WC, Williamson MR, et al. Effects of age, breast density, ethnicity, and estrogen replacement therapy on screening mammographic sensitivity and cancer stage at diagnosis: review of 183,134 screening mammograms in Albuquerque, New Mexico. Radiology. 1998;209:511-518.

4. Krecke KN, Gisvold JJ. Invasive lobular carcinoma of the breast: mammographic findings and extent of disease at diagnosis in 184 patients. AJR Am J Roentgenol. 1993;161:957-960.

5. Adejolu M, Krishnamurthy S, Whitman GJ. Ultrasound of invasive lobular carcinoma. Ultrasound Clin. 2011;6:313-325.

6. Butler RS, Venta LA, Wiley EL, Ellis RL, Dempsey PJ, Rubin E. Sonographic evaluation of infiltrating lobular carcinoma. AJR Am J Roentgenol. 1999;172(2):325-330.

7. Lopez JK, Bassett LW. Invasive lobular carcinoma of the breast: spectrum of mammographic, US, and MR imaging findings. Radio Graphics. 2009;29:165-176.

8. EvansWP, Warren Burhenne LJ, Laurie L, O'Shaughnessy KF, Castellino RA. Invasive lobular carcinoma of the breast: mammographic characteristics and computer-aided detection. Radiology. 2002;225: 182-189.

9. Le Gal M, Ollivier L, Asselain B, et al. Mammographic features of 455 invasive lobular carcinomas. Radiology. 1992;185:705-708.

10. Hilleren DJ, Andersson IT, Lindholm K, Linnell FS. Invasive lobular carcinoma: mammographic findings in a 10-year experience. Radiology. 1991;178:149-154.

11. Krecke KN, Gisvold JJ. Invasive lobular carcinoma of the breast: mammographic findings and extent of disease at diagnosis in 184 patients. AJR Am J Roentgenol. 1993;161:957-960.

12. Cornford EJ, Wilson AR, Athanassiou E, et al. Mammographic features of invasive lobular and invasive ductal carcinoma of the breast: a comparative analysis. Br J Radiol. 1995;68:450-453.

13. Qayyum A, Birdwell RL, Daniel BL, et al. MR imaging features of infiltrating lobular carcinoma of the breast histopathologic correlation. AJR Am J Roentgenol. 2002;178(5):1227-1232.

14. Selinko VL, Middleton LP, Dempsey PJ. Role of sonography in diagnosing and staging invasive lobular carcinoma. J Clin Ultrasound. 2004;32:323-332.

15. Rissanen T, Tikkakoski T, Autio A-L, Apaja-Sarkkinen M. Ultrasonography of invasive lobular breast carcinoma. Acta Radiol. 1998;39(3):285-291.

16. Berg WA, Gilbreath PL. Multicentric and multifocal cancer: wholebreast US in preoperative evaluation. Radiology. 2000;214:59-66.

17. Stafford RJ, Whitman GJ. Ultrasound physics and technology in breast imaging. Ultrasound Clin. 2011;6:299-312.

18. Schulz-Wendtland R, Bock K, Aichinger U, et al. Ultrasound examination of the breast with $7.5 \mathrm{MHz}$ and $13 \mathrm{MHz}$-transducers: scope for improving diagnostic accuracy in complementary breast diagnostics? Ultraschall Med. 2005;26:209-215.

19. Cawson JN, Law EM, Kavanagh AM. Invasive lobular carcinoma: sonographic features of cancers detected in a breast screen program. Australas Radiol. 2001;45:25-30.

20. Watermann DO, Tempfer C, Hefler LA, Parat C, Stickeler E. Ultrasound morphology of invasive lobular breast cancer is different compared with other types of breast cancer. Ultrasound Med Biol. 2005;31: 167-174.

21. Wilkinson LS, Given-Wilson R, Hall T, Potts H, Sharma AK, Smith E. Increasing the diagnosis of multifocal primary breast cancer by the use of bilateral whole-breast ultrasound. Clin Radiol. 2005; 60:573-578.

22. Moon WK, Im JG, Koh YH, Noh DY, Park IA. US of mammographically detected clustered microcalcifications. Radiology. 2000;217: 849-854.

23. Berg WA, Gutierrez L, NessAiver MS, et al. Diagnostic accuracy of mammography, clinical examination, US, and MR imaging in preoperative assessment of breast cancer. Radiology. 2004;233: $830-849$. 


\section{Publish your work in this journal}

Breast Cancer: Targets and Therapy is an international, peer- View the full aims and scopes of this journal here. The manuscript reviewed open access journal focusing on breast cancer research, identification of therapeutic targets and the optimal use of preventative and integrated treatment interventions to achieve improved outcomes, enhanced survival and quality of life for the cancer patient. management system is completely online and includes a very quick and fair peer-review system, which is all easy to use. Visit http:// www.dovepress.com/testimonials.php to read real quotes from published authors.

Submit your manuscript here: http://www.dovepress.com/breast-cancer---targets-and-therapy-journal 